\title{
Sentinel surveillance systems with special focus on vector-borne diseases
}

\author{
V. Racloz ${ }^{1}$, C. Griot ${ }^{2}$ and K. D. C. Stärk ${ }^{1 *}$ \\ ${ }^{1}$ Swiss Federal Veterinary Office, Schwarzenburgstrasse 155, 3003 Bern, Switzerland and \\ ${ }^{2}$ Institute of Virology and Immunoprophylaxis, Mittelhäusern, Switzerland
}

Received 8 June 2006; Accepted 18 July 2006

\begin{abstract}
In the past few decades, vector-borne diseases have been spreading into countries previously free of these agents. It is necessary for a surveillance method to be tailored to the biology of these agents in order to detect their incursion. Using a sentinel herd system, it is possible to target high-risk areas where occurrence is most probably due to vector presence. Since the 1970s, diseases such as Akabane, vesicular stomatitis and Bluetongue disease have successfully been monitored using cattle herds as sentinels in many countries such as Saudi Arabia, Australia, China, Indonesia, Sultanate of Oman and most recently in countries in Western Europe. This paper reviews the strengths and weaknesses of sentinel herd surveillance systems in general. In order to determine their efficacy, the following criteria were found to be essential: the choice of sentinel locations, sentinel animal, seasonality of sampling and diagnostic testing methods. We conclude that due to its ability to focus on a specific disease, sentinel herd systems have been successful in the early detection of the spread of a targeted agent. This review is used as a basis for recommendations for the development of future sentinel herd systems.
\end{abstract}

Keywords: vector-borne disease, sentinel herds, early warning, surveillance

\section{Introduction}

In order to maintain healthy wildlife and livestock populations, it is important to control and prevent diseases from occurring and spreading. Due to the variety of animal diseases circulating worldwide, it is important to have a disease monitoring and surveillance system tailored to the pathogen in question. Over the past few decades, vector-borne diseases have been emerging into geographic areas previously free of such infections. Malaria, Rift Valley fever (RVF), Dengue fever, Ross River virus, Murray Valley encephalitis, schistosomiasis and Japanese encephalitis are examples of diseases where factors such as climate change, intensification of agriculture and urbanization have led to an increased incidence, especially in developing countries (Sutherst, 2004). Other factors aiding this emergence include the expanding pattern of tourist travel, as well as animal movement

*Corresponding author. E-mail: katharina.staerk@bvet.admin.ch
(Anonymous, 2005a). This propagation is sustained by the fact that in the last decade, environmental changes through global warming have created new regions for vector-borne diseases to establish themselves (Gubler, 1998). Since these diseases were historically confined to other regions of the world, there is little evidence or experience on how the disease or its vector will behave in a new surrounding with different habitats, climates and susceptible hosts.

By studying livestock disease patterns and their dynamics, various surveillance strategies have been devised and applied. They share the aims of minimizing the effects of a disease upon a population, as well as preventing its spread to surrounding areas either within national borders or internationally. Animal disease surveillance has two main purposes, one of which is its use as a tool in assessing the health status of a population. This includes the detection of emerging exotic diseases and their vectors, as well as monitoring the shift in endemic disease prevalence. The second purpose is to determine the effectiveness of a specific control strategy 
that has been created to manage the disease, for example by routine sampling at slaughter houses in a national disease eradication program.

Surveillance is described as a systematic collection, analysis and interpretation of disease-related events occurring in a population, allowing for the implementation and planning of control measures subject to the results obtained from the surveillance system (Anonymous, 2004). Several types of surveillance methods exist, and are classified according to their function and data collection method (Salman, 2003; Thrusfield, 2005), yet the mainstay of this review will be sentinel surveillance. Passive surveillance is defined as a fixed, routine method which typically involves examining clinical cases, and usually relies on veterinarians and farmers to report suspicious cases. The disadvantages of passive surveillance are factors such as under-reporting and selection bias depending on the data source. Active surveillance can include the sampling of clinically normal animals and entails a more active and purposeful cooperation from stakeholders involved. Surveys, sentinel systems and mass screening methods are examples of active surveillance. Surveillance can also be categorized on the basis of the way the observation units are chosen: probability (random) or non-probability (non-random) sampling. Random sampling involves the selection of the sampling unit in as unbiased a manner as possible, so that each unit has an equal chance of being chosen. Non-random sampling includes strategies such as risk-based, targeted and sentinel surveillance. In these surveillance types, the sampling units are selected primarily by the investigator and are based on choosing a specific unit to suit the objective of the study.

The term 'sentinel' originates from the Latin word, sentire: to feel, and transformed itself into sentina: Italian for vigilance, and finally, into sentinelle in French. In epidemiological terms, a sentinel herd is defined as a cohort of animals at a pre-determined location, which is monitored over a specified period of time with respect to a specified disease agent (Ward et al., 1995). As explained by McCluskey, sentinel surveillance is used 'to monitor or identify outbreaks and epidemics caused by infectious agents, to investigate changes in prevalence or incidence of endemic or infectious agents, to evaluate the effectiveness of newly instituted disease control programs, and to confirm a hypothesis about the ecology or epidemiology of an infectious agent' (Salman, 2003). According to the World Animal Health Organization (OIE), sentinel units are described as groups of animals whose geographic location and immune status are known that are identified and regularly tested in order to detect disease occurrence. The data collected can provide information on the local incidence rate or prevalence as well as prove the freedom of infection status of the specific pathogen under investigation.

The establishment of a sentinel herd system allows for a targeted surveillance using risk-factor knowledge.
The term 'targeted surveillance' involves testing certain animals of a sub-population where disease is more likely to be introduced or found, and is part of a risk-based surveillance strategy. A proposed definition for risk-based surveillance is 'a surveillance programme in the design of which exposure and risk assessment methods have been applied together with traditional design approaches in order to assure appropriate and cost-effective data collection' (Stärk et al., 2006). It can be applied to a wide range of diseases and conditions such as animal welfare, endemic or exotic, infectious as well as vector-borne diseases.

This review aims to describe the various criteria used in selecting sentinel animals, location and sampling strategies involved for a variety of diseases, as well as determining the factors for assessing the efficacy of sentinel herd systems as a surveillance method. This has been accomplished through the collection of peerreviewed articles and studying documentation of national surveillance programs. Recommendations for use of sentinel herd surveillance and its success factors, as well as its disadvantages will also be discussed.

\section{Application}

Sentinel herd surveillance has been regularly used over the past few decades covering a wide variety of diseases and conditions. Studies using sentinel herds have been conducted for parasitic, viral, bacterial and vector-borne diseases as well as for toxicological screening and animal welfare issues. Examples of established sentinel herd surveillance programs are shown in Table 1. Although sentinel herd surveillance has been used for such a broad range of diseases, the basis of creating a sentinel herd as a surveillance tool remains similar in all cases. It consists of two parts: firstly, establishing the objective of the surveillance, and secondly, deciding upon the specific selection and design criteria needed depending on the nature of the disease/condition in question.

\section{Establishing the objective of the sentinel herd surveillance}

Whether a disease is endemic to a region or country, or is considered exotic, sentinel herd systems can be tailored specifically to the type of surveillance needed. Sentinel herd surveillance can be divided into two main branches (Fig. 1). Firstly, one branch involves measuring the frequency of an existing disease within different study objectives. Three main objectives within this group are: (1) to monitor the occurrence or to determine the dynamics of a specific disease, (2) to test a control strategy for a specific disease and (3) to assess exposure risk. The second branch of sentinel herd surveillance is its use as an early warning tool for either (1) detecting 
Table 1. Examples of sentinel herd surveillance programs

\begin{tabular}{llll}
\hline Disease or condition & Country & Sentinel animal & References \\
\hline Akabane disease & Saudi Arabia & Cattle, sheep, goat & Abu Elzein et al. (1998) \\
Air pollution & Canada & Cattle & Waldner et al. (2001) \\
Avian Influenza & France, Holland & Birds & EUROPA IP/06/210 \\
Bluetongue & Australia & Cattle & HAMP \\
Bovine dermatophilosis & USA & Cattle & Hadrill and Walker (1994) \\
Bovine ephemeral fever & Australia & Cattle & Seorge (1985) \\
Bovine viral diarrhea virus & Canada & Cattle & Waldner and Campbell (2005) \\
East Coast fever (Theileria) & Zambia & Cattle & Billiouw et al. (2005) \\
Epizootic hemorrhagic disease & Sudan & Cattle & Mohammed et al. (1996) \\
Internal parasites & New Zealand & Deer & Audige et al. (1998) \\
Livestock comfort & USA & Cattle & Cook et al. (2005) \\
Lyme disease & USA & Dog & Duncan et al. (2005) \\
Rift Valley fever & Africa & Sheep, goat & Chevalier et al. (2005) \\
St. Louis encephalitis & USA & Chicken & CDC \\
Trypanosomiasis & Burkina Faso & Cattle & Paling et al. (1987) \\
Vesicular stomatitis & USA & Horse & McCluskey et al. (2002) \\
West Nile & USA & Crow & Eidson et al. (2001) \\
Western equine encephalomyelitis & USA & Chicken & CDC \\
Xenotransplantation & USA & Pig & Iverson and Talbot (1998)
\end{tabular}

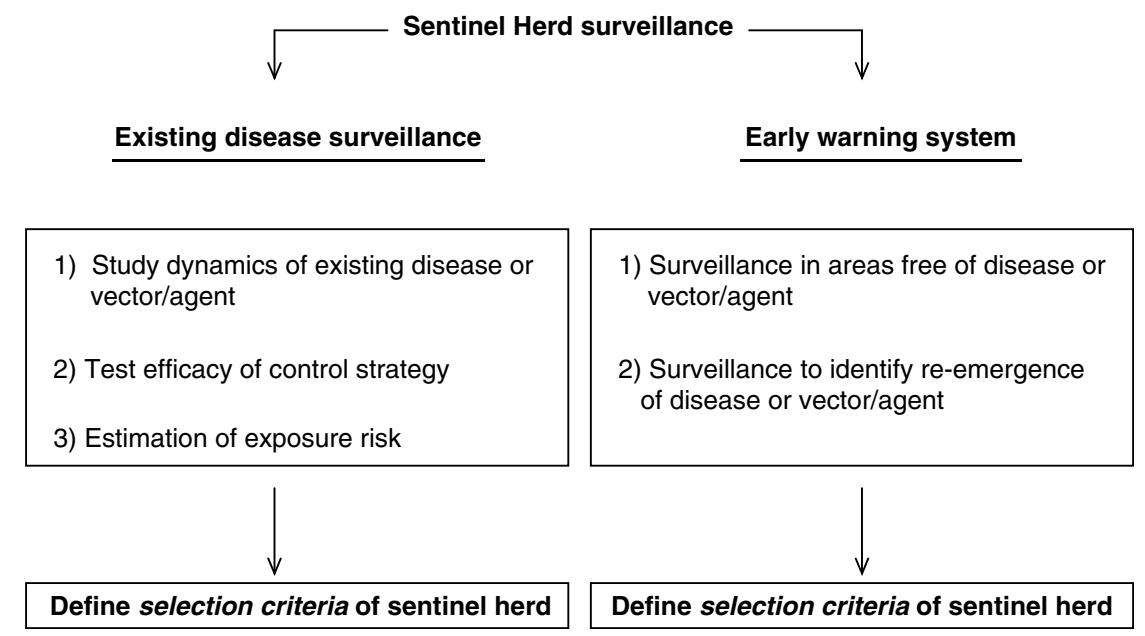

Fig. 1. Diagram of sentinel herd surveillance design. The design needs to take into consideration the objectives, and subsequent sentinel herd selection.

first incursion of a disease or its vector into previously free regions or (2) detecting its return to that area (Fig. 1).

Once the objective of the sentinel herd program has been established, design and selection criteria need to be defined in order to fit the disease, condition, vector or agent under investigation. This includes defining the selection of a specific region, sentinel animal species, characteristic of the herd, and finally determining the type and frequency of testing (Salman, 2003) (Fig. 2).

The choice of location largely depends on the actual purpose of the sentinel surveillance. If the main objective is the surveillance of an existing disease, this would imply choosing a location where the disease is known to circulate, and would therefore be non-random. If the disease is endemic, the choice of sentinel herd can be random. Furthermore, the sentinel animal within the herd can then be randomly chosen, granted it is susceptible to infection. If the goal of the surveillance is to serve as an early warning system, it is important to choose a location considered as a high-risk zone for incursion of the agent or vector. The identification of high-risk zones is essential since missing the first incursion of disease or its vector could have serious consequences for the rest of the country, as well as rendering the system ineffective. Vector-borne diseases occur in areas where the vector can establish itself, therefore high-risk zones can be identified depending on climatic and geographic factors as well as actual vector presence (e.g. determined by entomological trapping methods). The selection of the sentinel location is therefore non-random, although the choice of sentinel animal within this herd can eventually be random. Different regions represent varying levels 


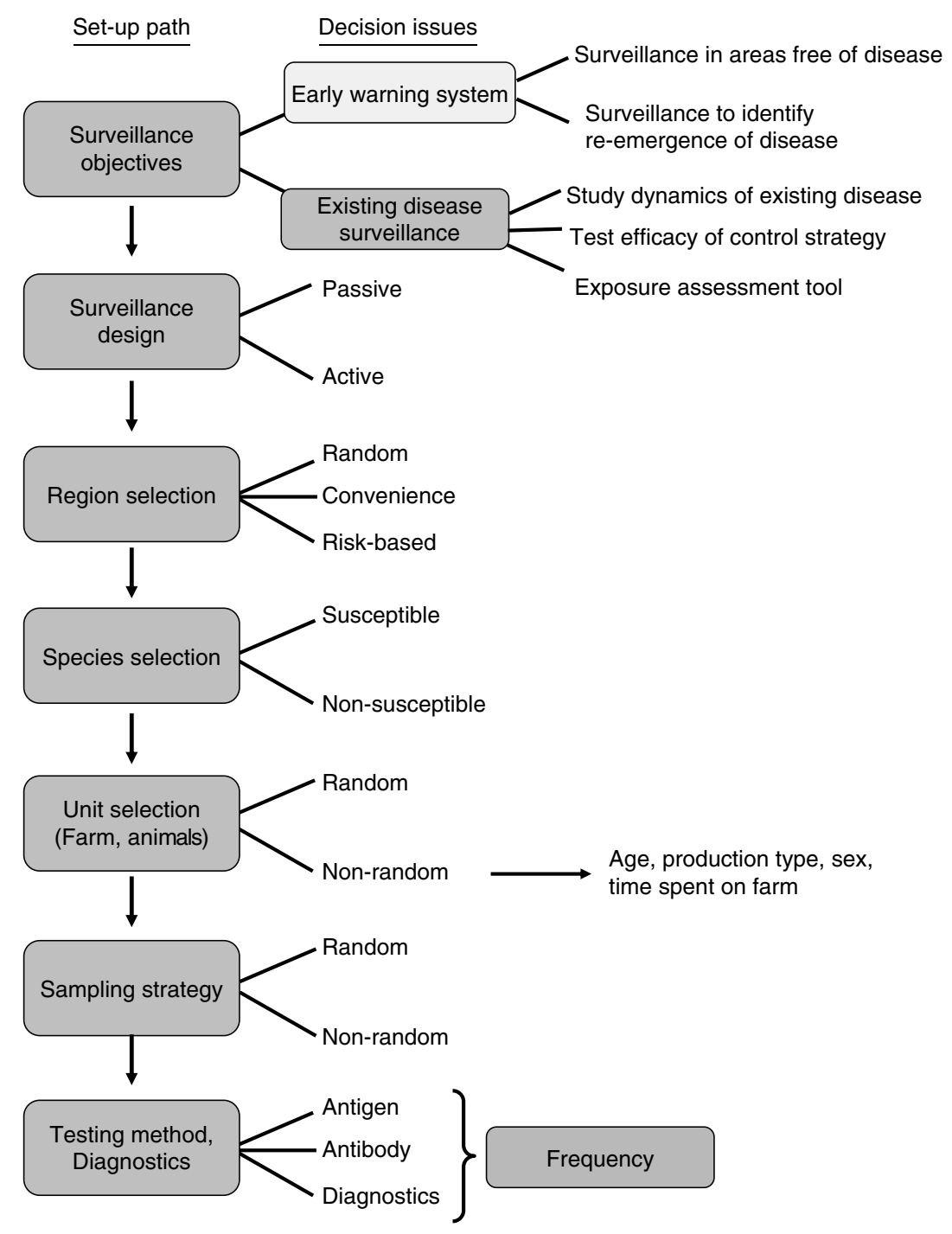

Fig. 2. Set-up path for establishment of a sentinel program. Establishment of a sentinel herd program requires the definition of study objective; selection of sentinel herd site, sentinel animal species and sentinel unit; development of a sampling strategy; and selection of diagnostic procedures.

of disease/vector exposure risk to the susceptible host population. Therefore, depending on the study objective, the decision on choice of herd location needs to take this into consideration. Exposure risks depend on a variety of factors including production purpose, or type of husbandry management in place (e.g. livestock kept on the same farm have different levels of exposure risk when compared to cattle displaced to alpine pastures during the summer months). In terms of vector-borne diseases, the exposure risk would be the time spent in areas where vectors are present.

Selecting the sentinel animal species requires epidemiological knowledge of the disease in question. As a minimal rule, the sentinel animal species must be susceptible to the disease and be able to generate a measurable response, preferably more easily detectable than in other species susceptible to the same disease. Selection criteria concerning the age of the sentinel animal vary depending on the aim of the surveillance. If the incidence of the disease is desired, one could designate younger animals as sentinels, since they have only been on the farm for fewer (vector) seasons, hence exposure is limited.

Depending on the seasonality, mode of transmission and severity of the disease, the testing period and diagnostic methods used will differ in each case. Surveillance of an exotic or highly pathogenic infection will need more frequent sampling, while testing for a vector-borne disease generally takes place before and/or after the vector season has occurred. The timing will depend on the epidemiology of the disease.

A particular difficulty for the establishment of an appropriate surveillance system concerning vector-borne diseases is that in addition to determining the objective of the surveillance and selecting the set-up criteria, the actual biology and consequent ecology of the vector 
have to be taken into consideration. This implies acquiring background knowledge on the geographical and climatic preferences of the vector and potential risk factors involved in terms of their occurrence and/or establishment into an area where susceptible hosts are located. Seasonal dynamics, landscape features, meteorological data and host preference are factors to be considered when selecting the location of the sentinel herd for a vector-borne disease. An additional challenge is that a certain agent might be transmitted by different vectors depending on the location, thereby resulting in variation in sentinel herd strategies for each specific country. For example, Bluetongue disease is transmitted by different species of the Culicoides midge depending on the country that is involved, hence various environmental conditions need to be addressed when planning to set up a sentinel herd for surveillance of this disease (Kline and Wood, 1988; Schmidtmann et al., 2000). It is therefore advisable for a surveillance system involving a vector-borne disease to be made of two components: a sentinel herd host surveillance, and an entomological surveillance activity. This will provide for determination of the vector species composition as well as monitoring the changes in frequency and abundance in order to establish certain patterns in their life cycles for that specific region (preferably near the sentinel herd location). In the United States, this type of setting (i.e. vertebrate host and vector surveillance) has been established for diseases such as West Nile (WN), Eastern and Western equine encephalitis, La Crosse and St. Louis encephalitis and Bluetongue disease (Gubler, 1998). The National Arbovirus Monitoring Program (NAMP) in Australia also uses this method for the surveillance of Akabane, Bluetongue and Bovine Ephemeral Fever agents and vectors (Anonymous, 2002).

\section{Application of sentinel herds in the context of surveillance of an existing disease}

\section{Monitoring the occurrence or dynamics of a disease}

Vesicular stomatitis (VS) is an economically important arboviral disease of livestock, especially in horse and cattle, although the exact reservoir host has not yet been identified. In an epidemiological study conducted in Colorado, the persistence of VS was determined in a 3-year-long project (McCluskey et al., 2002). Twenty sentinel locations in the state of Colorado, USA, were visited and clinical examinations as well as serological tests were carried out. The selection criteria for the location of the sentinel herd were based on the previous presence of VS on the site, as well as the voluntary participation of the owner. Horses, chosen non-randomly by the owner, were used as sentinel animal species since records show that the United States has been experiencing a greater number of clinical cases in horses as compared to cattle (McCluskey et al., 1999). A competitive enzyme-linked immunosorbent assay (ELISA) was chosen due to its financial advantages and its high level of sensitivity. The testing strategy was conceived to identify sero-conversions, and since VS antibodies only remain in circulation for about 45-60 days (McCluskey and Mumford, 2000), sentinel animals were tested 3-4 times per year.

Another study with the aim of determining the incidence of a disease was conducted in Saudi Arabia for Akabane disease (Abu Elzein et al., 1998). Sentinel locations were chosen again depending on the recorded presence of the disease and its vector. Sentinel species included cattle, sheep and goat, all known to be susceptible carriers of Akabane disease. Serological testing conducted at specific time points included blood sampling from dams just after parturition, and from their offspring at birth before suckling. These specific time points would provide information on the role of maternal antibodies in Akabane disease transmission. Therefore the selection criteria of location, species and test strategy for this sentinel herd system were also tailored to suit the study objective and the epidemiology of the disease in question.

\section{Testing the efficacy of a control strategy for a specific disease}

Substantial trade restrictions resulted from the presence of certain parasites in the deer industry in New Zealand. In this example, sentinel herd surveillance was used in order to evaluate different internal parasite control strategies (Audige et al., 1998). As in the case of studying the dynamics of a disease, the actual presence of the disease is required when testing a control strategy. Sentinel farm locations were chosen by convenience, but were able to represent the typical deer farming conditions in that area. Individual sentinel deer were randomly chosen by the research group to avoid farmer bias, and grouped by sex and age to receive anthelmintic treatments. The testing strategy involved blood sampling as well as counts of faecal egg and larvae collected in spring, summer, autumn and winter. This design allowed for the collection of samples throughout four different seasons as well as corresponding to deer reproduction patterns. The laboratory test used was the standard screening method for veterinary purpose available at that time.

A different approach involving the use of sentinel herds to test a control strategy was applied in a study to determine the effect of acaricide control on the island of Nevis in the Caribbean. A sentinel herd of cattle (Bos taurus) was used as a control group to determine the efficacy of acaricide treatment (in a group of similar cattle) for controlling bovine dermatophilosis (Hadrill and Walker, 1994). The choice of the sentinel species was supported by evidence that B. taurus are considered more susceptible to tick bites than other cattle breeds 
in similar geographic regions (Tatchell and Moorhouse, 1968). The testing procedure involved weekly counts of adult ticks and ranking clinical symptoms on the sentinels compared to the cattle undergoing acaricide treatment.

A similar approach was used to test treatments against African animal trypanosomiasis (AAT) in a study conducted in Burkina Faso (Bauer et al., 1992). The sentinel animals underwent initial treatment before the study was conducted and were used as a control group to measure the efficacy of flumethrin pour-on in cattle.

In contrast, a different approach to test the efficacy of a control strategy is the incorporation of sentinel animals into a vaccinated herd. An important factor to combat Avian Influenza is the ability to differentiate vaccinated poultry from infected poultry and birds. It was envisaged to apply this strategy recently in certain regions of France (the departments of Landes, Loire-Atlantique and Vendée), as part of a monitoring program. The aim of the vaccination was to reach 90,000 birds by the beginning of April 2006 and included a pre-vaccination examination of flocks to ensure health and bio-security standards, monthly clinical surveillance of the holdings, and finally the use of non-vaccinated sentinel birds to detect any outbreaks in the vaccinated flock. A similar method was planned for a Dutch vaccination campaign applied to hobby poultry and to free-range laying hens throughout the whole country (Anonymous, 2006).

\section{Sentinel herd used to estimate exposure risk}

Similarly to the two previous objectives, the selection of the sentinel region to assess exposure risk needs to be based on previous recorded disease presence. Lyme disease is found in many areas of Europe and the United States and represents one of the most reported tick-borne diseases in the latter country. Due to their behavior and close interaction with humans, dogs have been reported to be effective sentinel animals to determine human risk of Lyme disease in certain states of America (Duncan et al., 2005). Based on convenience sampling, canine serum samples were tested using a very specific and sensitive ELISA test. The aim of the study was to determine whether dogs could be used as a risk indicator of disease occurrence in the human population in similar geographic locations. Sentinel dogs originated from 'hotspot' states where previous history has shown a high occurrence of the disease, mostly in the eastern coast of the United States.

Another example of the use of a sentinel herd as an exposure assessment tool was conducted in a study to determine the risk associated with the possible transmission of diseases through xenotransplantation (Iverson and Talbot, 1998). Sentinel pigs were used to monitor herd health in order to ensure the absence of pathogens in any cells, tissues, or organs, that might potentially be transplanted into an immunosuppressed human recipient. Individual pigs were randomly chosen to be representative of their population including a variety of ages and both sexes. Direct contact between the sentinel and non-sentinel pigs in the herd (as well as with excreta) was important in order to maximize the potential exposure of the sentinels to any pathogens that may have been present (W. O. Iverson, personal communication).

\section{Application of sentinel herds as an early warning system}

\section{Surveillance in disease-free areas}

In disease-free areas, it is important to locate zones where the first incursion of the disease/vector is most probable, as opposed to endemic diseases whose presence has been extensively recorded. Bluetongue disease is a vector-borne animal disease of economic importance which has occurred in epidemic proportions since 1998 throughout the Mediterranean Basin and Western Europe (Purse et al., 2005). Due to its presence in countries adjacent to Switzerland, a nationwide serological survey was conducted in 2003, which involved the serological sampling of randomly selected cattle farms (Cagienard et al., 2004), as well as the establishment of entomological trapping at predetermined 'risk sites'. Risk sites were chosen mainly on the basis of climatic and geographic factors that limited vector biology, such as average yearly temperature and altitude respectively. Based on these results, sentinel herds were selected for annual serological sampling with the aim of creating an early warning system to detect the primary incursion of the agent before substantial spread could occur. Cattle were chosen as the target species primarily since they act as the reservoir species for the blue tongue virus (BTV) in sheep as well as presenting an earlier antibody response post-infection (Anonymous, 2004). Cattle farms are more abundant and more widely distributed in Switzerland and, finally, it has been shown that Culicoides midges have a feeding preference for cattle compared to sheep (Nevill et al., 1978).

Another use of sentinel herd surveillance as an early warning system is to prevent the spread of blue tongue (BT) disease into new areas such as the system used in Australia. Sentinel herds have been chosen to outline the 'possible activity' zones which cover all eight states (Anonymous, 2002). From 2003-2004, 84 sentinel cattle sites and 103 vector trapping sites were monitored. Through the focused surveillance of northern Australia, it allowed for the detection of new incursions as well as being an early warning tool for monitoring spread into southern areas especially since BTV has not occurred in major commercial sheep flocks.

\section{Surveillance to identify re-emergence of disease}

When the purpose of the sentinel surveillance is to serve as an early warning system for a disease which has already affected an area previously, the main function 
is to detect its re-emergence as rapidly as possible. Since 1999, WN virus has been recorded in the United States and is now considered endemic. In order to guide public health action in relation to the disease, the use of crow deaths as sentinels was recorded as an early warning system for human cases (Eidson et al., 2001; Anonymous, 2005c). Crows were used as sentinel animals, firstly by coincidence (other bird species were not studied in enough detail at this point), and secondly, since birds in general seem to be the major introductory or amplification route of this disease. Typical for studies involving wildlife, a convenience-based selection of sampling location was required. In this study, the crow death site determined the sentinel location choice. Most sightings were of individual birds as opposed to clusters which is more common in diseases such as Avian Influenza. The deaths were noted on a mapping system in order to visualize the pattern that developed. This sentinel system was useful as a possibility of determining the incursion of virus into areas as well as correlating them with human cases.

In aid of the prevention and control of RVF in western Africa, sentinel herd monitoring has been set up since the year 2000 (Anonymous, 2005b). Sentinel locations were selected based on geographically representative areas. This involved determining 'hot-spots' for vector activity and included areas near bodies of water such as rivers or swamps. This is in contrast to the previous study where sentinel location was random and not dictated by specific 'risk factors'. Sheep and goat were chosen as sentinel species, with the additional criteria of an age limit and preferred sex, due to the lessened probability of being slaughtered during the study. As in several other studies, sentinel animals which sero-converted during the study were replaced by sero-negative animals in order to detect any new infections. Blood sampling was the diagnostic method used with a regular frequency of testing. In relation to climate, the animals were sampled before and during the rainy season and it was recommended that the herd size be kept at a specific number in order to facilitate testing and eventual replacements.

\section{Merits and limitations}

In economic terms, a sentinel-herd-based surveillance can be an attractive option due to its targeted characteristic as opposed to a random surveillance system. It can detect individual cases since data collection is traceable and organized. Sentinel herd surveillance can either be passive or active depending on the objective in mind, and therefore provides flexibility for the participators involved. It has been shown to be an effective method as an early warning tool, and therefore can help to detect the initial entry or resurgence of a targeted disease/vector. Another merit of sentinel herd surveillance is the generally voluntary participation of farmers. The cooperation is often due to the possible exchange of information, allowing for a motivated partnership and a more successful follow-up procedure. Depending on the prevalence of the disease in a country, and the adopted sentinel surveillance strategy, it is possible to estimate and extrapolate morbidity measures/indicators for a wider population range. The main advantage of a sentinel herd system is its broad range of uses as well as its flexibility and focus.

In terms of validity, certain problems can arise, especially linked to the specific type of diagnostic test used. For example, certain immunological techniques will not be able to detect antibody presence after a certain period of time, and therefore the disease would pass unnoticed. Diagnostic tests involving antigen detection also have their limitations when considering the short circulation periods of certain agents, especially if visits to the sentinel herd are infrequent and clinical signs at the peak of the outbreak are missed, or if the sentinel species chosen is an asymptomatic carrier. The efficiency of diagnostic procedures may be low when the disease under investigation is uncommon to the area or seroprevalence is very low, whereby the specificity of the test will produce false positive results and generate a cascade of unnecessary actions. Depending on the method of sentinel animal or herd selection, data collected may present a biased finding, if it has not been carried out in a random fashion. If, on the other hand, a random selection process is decided upon, the location of the sentinel herd may not have been chosen for its accessibility (transport method), and a plan is needed to ensure that samples are sent and results are received in the quickest manner possible. Although voluntary participation has its advantages, it is also more difficult to force a farmer to remain in the study, and drop-outs, or lack of follow-ups can have an impact on the reliability of the sentinel herd system. Disease can also be missed upon incursion due to unstrategic sentinel herd locations.

\section{Discussion}

It is difficult to obtain specific information from sentinel herd literature using systematic search methods (Rabinowitz et al., 2005). Although the term 'sentinel herd' retrieved about 50 peer reviewed papers on the PUBMED search engine, actual information on selection criteria and specific details on use of sentinels was difficult to obtain for certain studies. This is mostly due to the omission of details when the design and selection criteria are described. Further searches through national programs and academic institutions were required to find a more comprehensive number of sentinel herd literature sources. The possibility of comparing sentinel herd systems with other systems with similar objectives was also difficult due to the lack of matching criteria 
listed in the studies. These problems were mostly overcome through personal communications with the authors who were very helpful by providing information upon request.

Based on the various objectives, the success of certain sentinel herd programs can be determined through the results obtained in the specific study. For a sentinel herd system belonging to the 'existing disease category', a study was conducted with the aim of calculating the background incidence level of RVF in Senegal (Chevalier et al., 2005). Sentinel animals were serologically tested and the results allowed for further strategic alterations as well as proving the efficacy of this type of surveillance system.

Measuring the effectiveness of a sentinel surveillance system in terms of an early warning tool is more difficult due to the lack of comparison points. Yet recently, the incorporation of models to assist in the design and selection of sentinel herds, or to forecast disease/vector occurrence has been increasing. Climate models were created in order to predict possible Bluetongue risk zones using temporal pattern data in a study conducted in Israel (Purse et al., 2004). Another example is the study by Giovannini et al., where in order to plan the continuation of the sentinel herd surveillance strategy in place since 2001 in Italy, a Monte Carlo model which simulated the expected number of sero-converting animals was created to help in decision-making (Giovannini et al., 2004).

Despite the limitations of sentinel herd surveillance systems, they can be very effective tools for the surveillance of specific conditions or disease categories. If the aims of the study are well defined, and the selection criteria have taken in all the specific disease/vector characteristics, setting up a sentinel unit as a targeted disease monitoring tool can be very successful. To date, sentinel herd surveillance has been used for a variety of purposes such as monitoring for the presence of new or re-emerging diseases, surveying anti-microbial resistance and even as a method to prevent bio-terrorism (Brannen et al., 2004). It is important to stress, however, that the successful use of sentinel herd surveillance depends on the precision of targeting a disease/vector, which inevitably depends on the availability and correct interpretation of epidemiological knowledge.

In this review, sentinel surveillance has been described for various objectives ranging from determining the dynamics of a specific disease, to measuring the efficacy of their control program, as well as an early warning system for emerging vector-borne diseases. The ability to tailor the surveillance by means of the particular selection of location, sentinel species and diagnostic method can create a valuable system for the detection of a wide range of diseases or conditions be it of welfare, endemic, exotic, infectious or vector-borne origin.

As mentioned in the merits and limitations of a sentinel surveillance system, due to the nature of sample collection and the infrastructure needed, sentinel herds are less suited for national disease prevalence surveys, and more are effective as early warning systems or for detection of the re-emergence of a disease.

Critical success factors exist more at the level of infrastructure and the availability of reliable disease and vector information, rather than at the data collection and data quality level. Especially concerning vector-borne diseases, the limited links between epidemiological, ecological and entomological data have made it difficult to allow for the full capacity of an early warning system to function at its highest potential. This has been improving in the last decade in large part due to improving technology and the use of satellite imagery in veterinary epidemiology as shown in various papers (Purse et al., 2004; Brownstein et al., 2005).

In conclusion, the success of a sentinel herd surveillance primarily depends on the purpose for which it was established, and more precisely on the actual establishment criteria regarding location, sentinel species and diagnostic methods. Detailed epidemiological knowledge of the agent under consideration is required to assure the adequate design of a sentinel surveillance program, and is therefore the most critical success factor.

\section{Acknowledgments}

We thank Drs Audige, Iverson, McCluskey and Mumford for the information they provided in aid of this literature research. This paper was financially supported by the Swiss Federal Veterinary Office.

\section{References}

Anonymous (2002). National Arbovirus Monitoring Program (NAMP). [Available online at www.namp.com.au.]

Anonymous (2003). Recognizing Rift Valley Fever. Animal Health Manual. Food and Agricultural Organization of the United Nations. [Available online at www.fao.org.]

Anonymous (2004). Weekly Disease Information and Handistatus II. World Organization for Animal Health (OIE). [Available online at www.oie.int.]

Anonymous (2005a). Infection, Science and Technology. The Economist 17 November 2005. [Available online at www. economist.com]

Anonymous (2005b). [Available online at www.fao.org.]

Anonymous (2005c). [Available online at www.cdc.gov.]

Anonymous (2006) European Union, EUROPA IP/06/210 Avian Influenza: vaccination of certain poultry in France and Netherlands approved. [Available online at www.europa. eu.int.]

Abu Elzein EM, al-Afaleq AI, Mellor PS, el-Bashir AM and Hassanein MM (1998). Study of Akabane infection in Saudi Arabia by the use of sentinel ruminants. Journal of Comparative Pathology 119: 473-478.

Audige LJ, Wilson PR and Morris RS (1998). A survey of internal parasites and parasite control on North Island deer farms. New Zealand Veterinary Journal 46: 203-215.

Bauer B, Kabore I, Liebisch A, Meyer F and Petrich-Bauer J (1992). Simultaneous control of ticks and tsetse flies in Satiri, Burkina Faso, by the use of flumethrin pour on for cattle. Tropical Medicine and Parasitology 43: 41-46. 
Billiouw M, Brandt J, Vercruysse J, Speybroeck N, Marcotty T, Mulumba M and Berkvens D (2005). Evaluation of the indirect fluorescent antibody test as a diagnostic tool for East Coast fever in eastern Zambia. Veterinary Parasitology 127: 189-198.

Brannen DE and Stanley SAR (2004). Critical issues in bioterrorism preparedness: before and after September 2001. Journal of Public Health Management and Practice 4: 290-298.

Brownstein JS, Skelly DK, Holford TR and Fish D (2005). Forest fragmentation predicts local scale heterogeneity of Lyme disease risk. Oecologia 146: 469-475.

Cagienard A, Dall'Acqua F, Thür B, Mellor PS, Denison E, Griot C and Stärk KDC (2004). Bluetongue surveillance in Switzerland in 2003. Veterinaria Italiana 40: 133-136.

Chevalier V, Lancelot R, Thiongane Y, Sall B, Diaite A and Mondet B (2005). Rift Valley fever in small ruminants, Senegal, 2003. Emerging Infectious Diseases 11: 1693-1700.

Cook NB, Bennett TB and Nordlund KV (2005). Monitoring indices of cow comfort in free-stall-housed dairy herds. Journal of Dairy Science 88: 3876-3885.

Duncan AW, Correa MT, Levine JF and Breitschwerdt EB (2005). The dog as a sentinel for human infection: prevalence of Borrelia burgdorferi C6 antibodies in dogs from southeastern and mid-Atlantic States. Vector-borne Zoonotic Diseases 5: 101-109.

Eidson M, Komar N, Sorhage F, Nelson R, Talbot T, Mostashari F and McLean R (2001). Crow deaths as a sentinel surveillance system for West Nile virus in the northeastern United States, 1999. Emerging Infectious Diseases 7: 615-620.

Giovannini A, MacDiarmid S, Calistri P, Conte A, Savini L, Nannini D and Weber S (2004). The use of risk assessment to decide the control strategy for bluetongue in Italian ruminant populations. Risk Analysis 24: 1737-1753.

Gubler DJ (1998). Resurgent vector-borne diseases as a global health problem. Emerging Infectious Diseases 4: 442-450.

Hadrill DJ and Walker AR (1994). Effect of acaricide control of Amblyomma variegatum ticks on bovine dermatophilosis on nevis. Tropical Animal Health and Production 26: $28-34$.

Iverson WO and Talbot T (1998). Definition of a production specification for xenotransplantation. A European perspective. Annals of the New York Academy of Sciences 862: 121-124.

Kline DL and Wood JR (1988). Habitat preference of coastal Culicoides spp. at Yankeetown, Florida. Journal of the American Mosquito Control Association 4: 456-465.

McCluskey BJ and Mumford EL (2000). Vesicular stomatitis and other vesicular, erosive, and ulcerative diseases of horses. Veterinary Clinics of North America: Equine Practice 16: 457-469.

McCluskey BJ, Hurd HS and Mumford EL (1999). Review of the 1997 outbreak of vesicular stomatitis in the western United States. Journal of the American Veterinary Medical Association 215: 1259-1262.

McCluskey BJ, Mumford EL, Salman MD and Traub-Dargatz JJ (2002). Use of sentinel herds to study the epidemiology of vesicular stomatitis in the state of Colorado. Annals of the New York Academy of Sciences 969: 205-209.

Mohammed ME, Aradaib IE, Mukhtar MM, Ghalib HW, Riemann HP, Oyejide A and Osburn BI (1996). Application of molecular biological techniques for detection of epizootic hemorrhagic disease virus (EHDV-318) recovered from a sentinel calf in central Sudan. Veterinary Microbiology 52: 201-208.

Nevill EM (1978). The use of cattle to protect sheep from bluetongue infection. Journal of the South African Veterinary Association 49: 129-130.

Paling RW, Leak SG, Katende J, Kamunya G and Moloo SK (1987). Epidemiology of animal trypanosomiasis on a cattle ranch in Kilifi, Kenya. Acta Tropica 44: 67-82.

Purse BV, Baylis M, Tatem AJ, Rogers DJ, Mellor PS, Van Ham M, Chizov-Ginzburg A and Braverman Y (2004). Predicting the risk of bluetongue through time: climate models of temporal patterns of outbreaks in Israel. Revue Scientifique et Technique de l'Office International des Epizooties 23: 761-775.

Purse BV, Mellor PS, Rogers DJ, Samuel AR, Mertens PP and Baylis M (2005). Climate change and the recent emergence of bluetongue in Europe. Nature Reviews: Microbiology 3: 171-181.

Rabinowitz P, Gordon Z, Holmes R, Taylor B, Wilcox M, Chudnov D, Nadkarni P and Dein FJ (2005). Animals as sentinels of human environmental health hazards: an evidence-based analysis. EcoHealth Journal Consortium 2: 26-37.

Salman MD (2003). Animal Disease Surveillance and Survey Systems. Ames, IA: Iowa State Press.

Schmidtmann ET, Bobian RJ and Belden RP (2000). Soil chemistries define aquatic habitats with immature populations of the Culicoides varipennis complex (Diptera: Ceratopogonidae). Journal of Medical Entomology 37: 58-64.

St George TD (1985). Studies on the pathogenesis of bovine ephemeral fever in sentinel cattle. I. Virology and serology. Veterinary Microbiology 10: 493-504.

Stärk KDC, Regula G, Hernandez J, Knopf L, Fuchs K, Morris RS and Davies P (2006). Concepts for risk-based surveillance in the field of veterinary medicine and veterinary public health: review of current approaches. BioMed Central Health Services Research 6: 20-28.

Sutherst RW (2004). Global change and human vulnerability to vector-borne diseases. Clinical Microbiology Reviews 17: $136-173$.

Tatchell RJ and Moorhouse DE (1968). The feeding processes of the cattle tick Boophilus microplus (Canestrini). II. The sequence of host-tissue changes. Parasitology 58: 441-459.

Thrusfield M (2005). Veterinary Epidemiology, 3rd edn. Ames, IA: Blackwell Science Ltd.

Waldner CL and Campbell JR (2005). Use of serologic evaluation for antibodies against bovine viral diarrhea virus for detection of persistently infected calves in beef herds. American Journal of Veterinary Research 66: 825-834.

Waldner CL, Ribble CS, Janzen ED and Campbell JR (2001). Associations between total sulfation, hydrogen sulfide deposition, and beef-cattle breeding outcomes in western Canada. Preventive Veterinary Medicine 50: 19-33.

Ward MP, Flanagan M, Carpenter TE, Hird DW, Thurmond MC, Johnson SJ and Dashorst ME (1995). Infection of cattle with bluetongue viruses in Queensland, Australia: results of a sentinel herd study, 1990-1992. Veterinary Microbiology 45 : 35-44. 